\title{
Self-Organized Energy Efficient Channel Assignment for Cognitive D2D Communication in $5 \mathrm{G}$ Networks
}

\author{
Najam ul Hasan*, Waleed Ejaz ${ }^{\dagger}$, Adnan Shahid ${ }^{\ddagger}$, Imran Baig*, Manaf Zghaibeh* \\ ${ }^{*}$ Department of Electrical and Computer Engineering, Dhofar University, Salalah, Oman \\ ${ }^{\dagger}$ Department of Electrical Engineering, Sultan Qaboos University, Muscat, Oman \\ $\ddagger$ IDLab, Department of Information Technology, Ghent University - imec, Belgium \\ Email: * nulhasan@du.edu.om
}

\begin{abstract}
With the rapid increase in the number of smartphones and bandwidth hungry applications, it is much more needed to use the bandwidth and power in an efficient manner. The 5G networks can fulfill these ramping demands of bandwidth and higher data rates by leveraging the device to device (D2D) communication. The D2D communication makes efficient use of the available resources that is bandwidth and power with the help of direct communication of the devices with each other without/partial contribution of the base station (BS). In this paper, first we defined an energy efficient channel assignment problem for a D2D network taking into account both the intra and inter link interference for D2D communication. Then, proposed a fully distributed game theory based solution to solve this problem, keeping in view the scalability and self-organization concerns of D2D communication. Finally, an exhaustive simulation is done to evaluate the proposed solution and results in presented in terms of energy efficiency in comparison with the well-known random, greedy approaches.
\end{abstract}

Index Terms - cognitive radio network, wireless mesh network, channel assignment, spectrum sharing.

\section{INTRODUCTION}

In currently employed $4 \mathrm{G}$ networks all the devices communicate via Base Station (BS) even if these devices are in very close vicinity. Although this mode of operation eases the network management but lacks in the efficient use of network resources mainly the bandwidth and the power [1,2]. Therefore, a new idea of D2D communication has emerged in the recent years for the upcoming $5 \mathrm{G}$ networks. It is anticipated that $5 \mathrm{G}$ cellular networks will be a combination of currently deployed cellular network along with a coexisting D2D networks as shown in Figure 1. This latest trends and increased interest in D2D communication is due to change in the attitude of the telecom operators. Previously, telecom operators believe that local services are just a fraction of overall communication services. However, by witnessing the recent growth in the number of context-aware services and applications, several vendors and operators deem D2D as a promising tool to reduce the cost of local services in terms of bandwidth and power consumption [3]. In D2D network the devices are allowed to communicate either directly or with the participation of BS. The Having a look at different existing

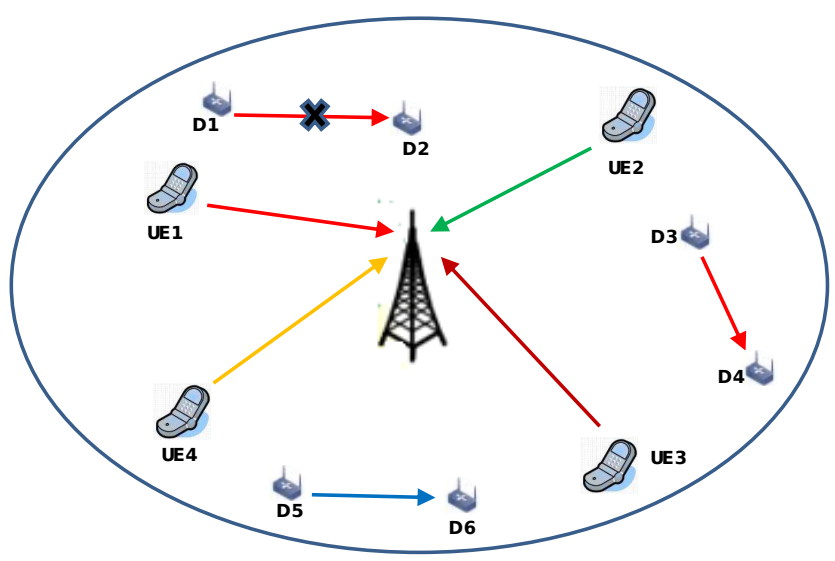

Fig. 1. Two tier Network: Macrocell and device tiers.

technologies, Wi-Fi and Bluetooth are already providing D2D communication. So the question is why we need to add D2D communication as a part of cellular communication network. The reason is that unlike Wi-Fi and Bluetooth which operates on the unlicensed spectrum, by adding D2D will allow devices to communicate with each other directly over the licensed channel. This will guarantees higher quality of service (QoS) and secure communication [4].

The basic idea of upcoming $5 \mathrm{G}$ networks is to operate as a two tiers networks i.e., macrocell and device tiers In macro-cell tier, device will communicate with other device through the BS. The device tier will implement the D2D communications. When devices communicate through base station (BS) they are said to be in the microcell tier. If two devices directly communicate with each other or realize their communication by relaying their information through other devices then these devices are said to be in device tier. The BSs will continue to serve the devices as usual in such a system. However, the devices which are at the cell edges or congested areas will be allowed to communicate in device tier 
which in turn will create an ad hoc mesh network. In devicetier communication, macrocell and device tiers, the operator might have different levels of control., , The operator may exercise full, partial or no control of BS over the allocated resources for the ongoing D2D communication. Based on this, D2D communication in device tier can be classified mainly in two different types [4].

Direct D2D Communication with Operator Controlled Link Establishment (DC-OC): In this model, BS is responsible to allocate the resources for D2D communication and it establishes the link between source and destination devices. However, after the link is established, communication can occur between the devices without going through BS.

Direct D2D Communication with Device Controlled Link Establishment (DC-DC): In this type of communication, BS is neither involved in allocation of resources nor in the link establishment. The devices are allowed to select the resources and communicate between them directly.

This paper opted DC-DC model for communication in the device tier. This is mainly focused towards a self-organized energy efficient channel assignment for D2D communication, while guaranting the desired communication QoS. The energy efficiency of a link is computed as a ratio of throughput obtained and energy consumed in an observation period. Therefore, we first established a link capacity and energy model based on PU activities, and then formulated an optimization problem for channel assignment in D2D communication, with the objective of maximizing the energy efficiency while considering the spectrum sharing constraints among the nodes. Then, to solve the problem while keeping in view the selforganization and scalability concerns of D2D communication, and simplicity of the solution to be implemented in realtime, we proposed the game theory based channel assignment approach.

The rest of the paper is organized as follows. Section 2 presents a system model problem description. Section 3 presents the methods to solve the formulated problem using game-theory Section 4 demonstrates simulation results and finally, conclusions are drawn in Section 5.

\section{System Model AND PROBlem Formulation}

The core objective of this paper is to develop a channel assignment mechanism for D2D communication in 5G networks by maximizing the overall energy efficiency of the network. It is assumed that all the D2D devices are equipped with cognitive radio capabilities. Therefore, each device has the capability of accessing the channels in an opportunistic manner. The energy efficiency $\eta$ of a link is defined as bits per joule, which serves as a metric for measuring the energy efficiency performance of the network and mathematically, it is written as,

$$
\eta=R / E
$$

where $R$ denotes the capacity of the link in the form of bits per second and $E$ is the energy consumed of the link per sec, respectively.

The number of bits transmitted bits over a link can be computed as follow,

$$
R=B \times T_{s}
$$

where $B$ is the throughput of the link and $T_{s}$ is the duration of the slot for which the channel assignment is performed. The throughput $B$ is computed using the Shannon's capacity formula, whereas, $T_{s}$ is a predefined duration. The energy consumed over a link can be computed as follows.

$$
E=P \times T_{x}+P_{i}\left[T_{s}-T_{x}\right]
$$

where $P$ and $P_{\text {idle }}$ are the power consumed by a device in transmitting or idle state respectively, and $T_{x}$ is the duration of transmission of a device during a slot duration $T_{s}$.

Given the $N$ number of devices, each with different available channels out of the $M$ total number of channels. The availability of each channel is modelled as an $O N-O F F$ poisson process [6]. Specifically,each device is allowed to access the available channel only when it is empty. Let $x_{n m}^{k}$ be the decision variable. The value of the decision variable $x_{n m}^{k}$ is 1 if the $k$ th channel is used for communication between the $n$th and the $m$ th $\mathrm{D} 2 \mathrm{D}$ devices, and 0 otherwise. Moreover, the $n$th and the $m$ th devices can communicate only if the $k_{t h}$ channel is empty for both of them and also if they are within the transmission range of each other. The channel assignment problem for D2D communication can be written in the form of an optimization problem as follows

$$
\begin{gathered}
\text { Maximize: } \sum_{k=1}^{K} \eta_{n m}^{k} x_{n m}^{k}, \forall n, m, \text { and } n \neq m . \\
C 1: \sum_{k} x_{n m}^{k}=1, \forall n, m, \text { and } n \neq m, \\
C 2: R_{n} \geq R_{M I N}, \\
C 3: D_{n m} \leq D_{M I N} .
\end{gathered}
$$

The objective of the above mentioned problem is to maximize the energy efficiency of D2D communication in a noncooperative manner until a Nash Equilibrium point is reached. The constraint $C 1$ means that only one channel is allocated for a link connecting the $m$ th and $n$th D2D devices. The constraint $C 2$ ensures that the minimum capacity requirement $R_{M I N}$ is maintained while enhancing the energy energy efficiency. The constraint $D_{n m}$ means that communication is only possible if the distance between the $m$ th and the $n$th devices $D_{n m}$ is within the communication range i.e., $D_{M I N}$.

\section{GAME THEORY FOR CHANNEL ASSIGNMENT}

\section{A. Game-theoretic framework}

In this study, we model the channel allocation for D2D 
communication as a strategic form non-cooperative game. Generally, a strategic form game is comprised of three main components: players, actions, and a utility function. Mathematically, a strategic form game is represented as $\nabla=$ $\left\{N,\left\{A_{n}\right\}_{n \in N},\left\{U_{n}(.)\right\}_{n \in N}\right\}$, where $N$ is a finite set of players (i.e, D2D devices), $A_{n}$ is a set of actions (i.e, channel allocation), and $U_{n}$ is a utility function which is associated as a reward for each player such that $U_{n}: A \rightarrow R$. A utility function is the satisfaction which is perceived by each $n$th player as a function of its chosen action $a_{n}$ and also the actions of other $N-1$ players such that $A_{n}=$ $\left\{A_{1}, A_{2}, \ldots, A_{n-1}, A_{n+1}, \ldots, A_{N}\right\}$.

\section{B. Potential game formulation}

In the proposed non-cooperative game, the action profile of each player is represented as $A_{n}=\Omega_{n}^{D 2 D}$, where $\Omega_{n}^{D 2 D}$ is an allocation of subset of channels from a total pool of $M$ channels. More specifically, each player makes decisions iteratively in a distributive manner until a Nash Equilibrium is reached. A Nash Equilibrium is defined as a stable operating point of a non-cooperative game and after that none of the players can change their actions unilaterally by increasing the utility function. A potential game converges to a Nash Equilibrium if better response dynamics is applied, where the best response dynamics is updating actions of the $n$th player while the actions of other players remains fixed [5]. To obtain the Nash equilibrium for the potential game several learning techniques are available. We opted the best response dynamic . Best response dynamics is a method of updating strategy fin which players maximize their individual utility function keeping the strategies of the other players constant. After traversing through all the players, if there is no more increase in the network utility is reported, then it mean a Nash equilibrium is achieved. Mathematically, the best response dynamics of the $n$th player associated with the action profile at time $t+1$ should satisfy the condition,

$$
A_{n}^{t+1}=\arg \max _{A_{n}^{\prime} \in A_{n}} U_{n}\left(A_{n}^{\prime}, A_{-n}^{t}\right)
$$

where $\left(A_{n}^{\prime}, A_{-n}^{t}\right) \epsilon A_{n}$ is the action profile of the $n$th player at time $t$. A strategic form non-cooperative game is termed as an exact potential game, if there exists a function $U_{n}$ such that,

$$
\Upsilon\left(A_{n}^{\prime}, A_{n}\right)-\Upsilon\left(A_{n}, A_{n}\right)=U_{n}\left(A_{n}^{\prime}, A_{-n}\right)-U_{n}\left(A_{n}, A_{-n}\right)
$$

The potential function in (6) represents that an improvement in the utility function corresponds to an improvement in the potential function.

\section{Proposed utility function and potential function}

To solve the problem given in equation (4) using a potential game approach, the utility function of the $n$th player is the same as the objective function,

$$
U_{n}\left(A_{n}, A_{-n}\right)=\sum_{k=1}^{K} \eta_{n}^{k},
$$

where the utility function $U_{n}$ mentioned above corresponds to the maximization of the energy efficiency of D2D communication.

Based upon the utility function in (10), the potential function is formulated as according to (9),

$$
\Upsilon_{n}\left(A_{n}, A_{-n}\right)=\sum_{n=1}^{N} \sum_{k=1}^{K} \eta_{n}^{k},
$$

Thus, if the afore-mentioned function is an exact potential function and $A^{*}=\operatorname{argmax}_{A \epsilon A_{n}} \Upsilon(A)$ is the maximization of the potential function, then the Nash Equilibrium is guranteed. On the other, the action profile is compact and convex, and the potential function $\Upsilon\left(A_{n}, A_{-n}\right)$ is continuously differentiable, the existence of a Nash Equilibrium is confirmed [7].

\section{Simulation Results}

In this section, first, we define the parameters chosen for the evaluation of the proposed technique. A geographical area of $500 \mathrm{~m}^{2}$ is considered for the deployment of D2D communication devices.. In the considered area, the devise are distributed in a random manner. It is assumed that each device has only two radio interfaces. $M$ unique channels are considered where each channel has a frequency band of $10 \mathrm{MHz}$. The channels available that are available for each device is a subset of $\mathrm{M}$ channels. And the channels which are available at any two devices may be partially of completely different from each other. In the simulation, this is done at each device by selecting some of the channels from the pool of these available $\mathrm{M}$ channels based on of the probability of the presence of absence of cellular user on that channel. The probability that a channel is empty or occupied is taken to be same for every channel. But it is independent for each channel. For simulation the probability that a channel is occupied is taken to be 0.8 . It is assumed that each device adopts binary orthogonal frequency shift keying modulation with a path- loss exponent $n$ equals to 4. Moreover, each device transmission range is 100 meters and the interference range is 150 meters with an antenna constant of 62.5 . The noise power spectral density value is taken to be $-110 \mathrm{dBm} / \mathrm{Hz}$. Furthermore, it is assumed that all the devices always have the data to be transmitted. To measure the the performance of the proposed technique, energy efficiency per node (bits/Joule/node) is considered as the performance metric, that is computed by varying the number of devices and channels.

Fig. 2 shows the average energy efficiency per device in terms of varying number of channels. The channels are varied from 10 to 25 . The numbers of devices are fixed to 100 . An increase in the available channels results in an increased opportunity of assigning channels to more and more devices. Therefore, the average energy efficiency per device increases 


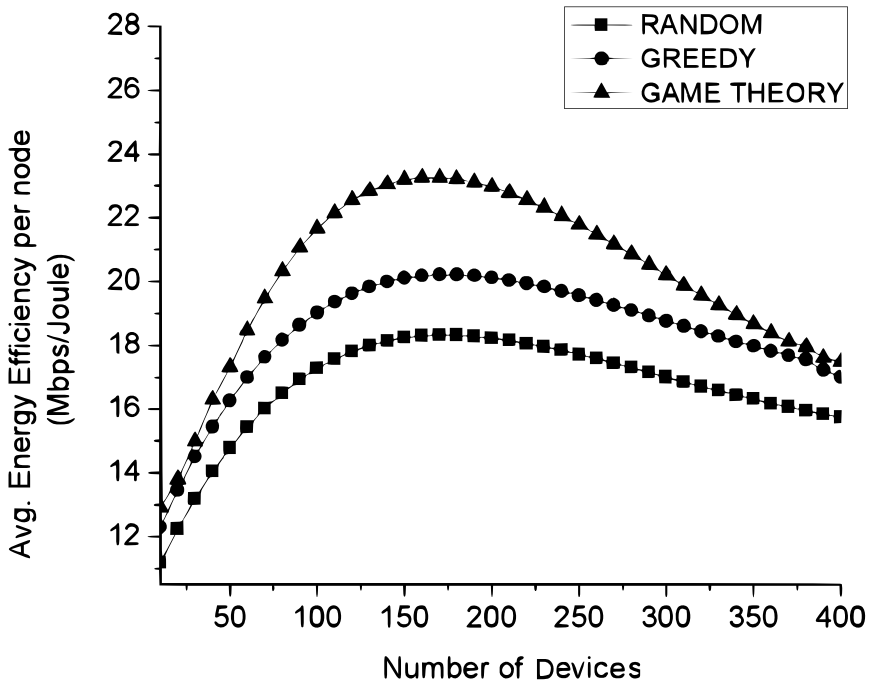

Fig. 2. Energy Efficiency versus number of devices.

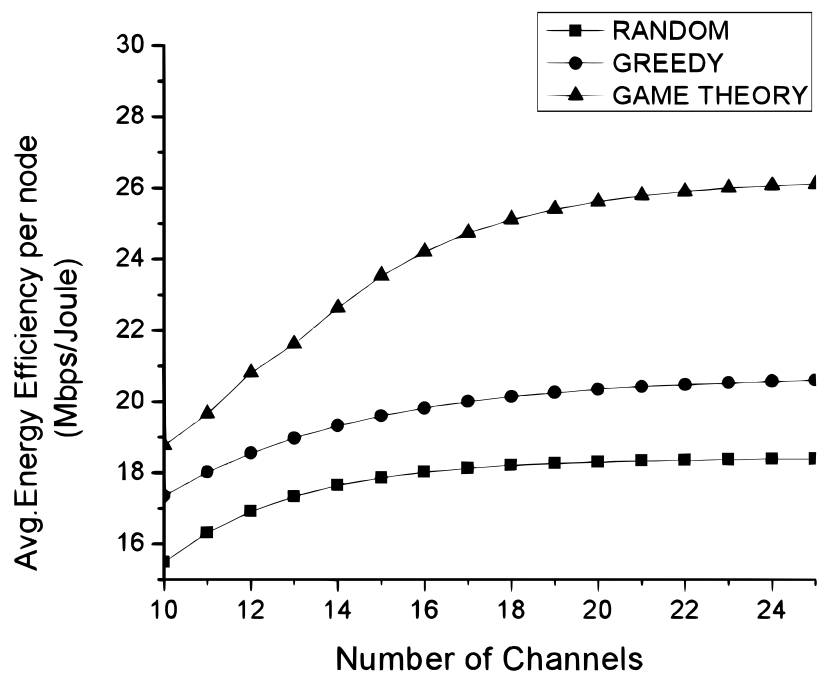

Fig. 3. Energy Efficiency versus number of Channels

with the increasing number of channels. But the rate slows down beyond a certain point for each technique because of the limit imposed on the number channel that can be assigned to each device as mentioned in constraint given in equation (4).

Fig. 3, shows the the average energy efficiency per device is reported in terms devices. The numbers of devices are varied from 10 to 400 . Initially, it can be seen that with increase in devices the average energy efficiency per device increases because of the room available to make efficient reuse of the available channels in the spatial domain. However, when devices are increased to a certain point in the considered geographical region, the average energy efficiency per device starts decreasing due to the fact that there is almost no more room left for spatial reuse of the available channels. Hence, average energy efficiency per device decreases with the increase in the number of devices.

\section{CONClusion}

In this paper, first, we discussed the motivation behind Channel assignment for cognitive radio based D2D communication. Then, we identified models for D2D communication. For this paper, we opted direct D2D communication model with assumption that each device is equipped with cognitive radio capability. Based on a cognitive radio based direct D2D communication model, an optimization problem is formulated for channel assignment. To solve this problem a fully distributed game theoretical approach. The objective for the proposed technique is to maximize the overall energy efficiency of the D2D communication devices. The simulation is done to evaluate the performance of the proposed technique in terms of energy efficiency by varying the number of devices and the number of channels. The simulation results show that the proposed approach is quite effective in assigning the channels to each link in terms energy efficiency.

\section{REFERENCES}

[1] A. Asadi, Q. Wang, and V.Mancuso, "A survey on device to device communication in cellular network," IEEE Commun. Surveys Tuts., vol. 16, no. 4, pp. 1801-1819, 2014.

[2] P. Gandotra and R. K. Jha, "Device-to-device communication in cellular networks: A survey," Journal of Network and Computer Applications., vol. 50, no. 13, pp. 2127-2159, 2016.

[3] D. Astely, E. Dahlman, G. Fodor, S. Parkvall, and J. Sachs, "Lte release 12 and beyond," IEEE Communication Magazine., vol. 50, no. 13, pp. 2127-2159, 2016.

[4] M. N. Tehrani, M. Uysal, and H. Yanikomeroglu," "Device-to-device communication in $5 \mathrm{~g}$ cellular networks: challenges, solutions, and future directions,' IEEE Communication Magazine., vol. 50, no. 13, pp. 2127 2159, 2016.

[5] M. Voorneveld,, "Best-response potential games. IEEE Communication Magazine., vol. 50, no. 13, pp. 2127-2159, 2016.

[6] I. Kaj, Aspects of wireless network modeling based on Poisson point processes. Fields Institute Workshop on Applied Probability, Carleton University, 2006.

[7] G. Scutari, S. Barbarossa, DP. Palomar, 'Potential games: A framework for vector power control problems with coupled constraints' InAcoustics, Speech and Signal Processing, 2006. ICASSP 2006 Proceedings. IEEE International Conference on May 14 (Vol. 4, pp. IV-IV), 2006. 